\title{
CLASSIFICATION OF FINITE-DIMENSIONAL TRIANGULAR HOPF ALGEBRAS WITH THE CHEVALLEY PROPERTY
}

\author{
Pavel Etingof and Shlomo Gelaki
}

\section{Introduction}

Recall $[\mathrm{AEG}]$ that a triangular Hopf algebra $A$ over $\mathbf{C}$ is said to have the Chevalley property if the tensor product of any two simple $A$-modules is semisimple, or, equivalently, if the radical of $A$ is a Hopf ideal. There are two reasons to study this class of triangular Hopf algebras: First, it contains all known examples of finite-dimensional triangular Hopf algebras; second, it can be, in a sense, "completely understood". Namely, it was shown in [AEG] that any finitedimensional triangular Hopf algebra with the Chevalley property is obtained by twisting of a finite-dimensional triangular Hopf algebra with $R$-matrix of rank $\leq 2$, which, in turn, is obtained by "modifying" the group algebra of a finite supergroup. This provides a classification of such Hopf algebras.

The goal of this paper is to make this classification more effective and explicit, i.e. to parameterize isomorphism classes of finite-dimensional triangular Hopf algebras with the Chevalley property by group-theoretical objects, similarly to how it was done in [EG2] in the semisimple case. This is achieved in Theorem 2.2 , where these classes are put in bijection with certain septuples of data. In the semisimple case, the septuples reduce to the quadruples of [EG2], and we recover the result of [EG2]. In the minimal triangular pointed case, we recover Theorem 5.1 of $[\mathrm{G}]$.

\section{The main theorem}

In this section we give an explicit description of the set of isomorphism classes of finite-dimensional triangular Hopf algebras over $\mathbf{C}$ with the Chevalley property. We will freely use the facts from the theory of Hopf superalgebras and finite supergroups which were sketched in [AEG].

Definition 2.1. A triangular septuple is a septuple $(G, W, H, Y, B, V, u)$ where $G$ is a finite group, $W$ is a finite-dimensional representation of $G, H$ is a subgroup of $G, Y$ is an $H$-invariant subspace of $W, B$ is an $H$-invariant nondegenerate element in $S^{2} Y, V$ is an irreducible projective representation of $H$ of dimension $|H|^{1 / 2}$, and $u \in G$ is a central element of order $\leq 2$ acting by -1 on $W$.

Received January 6, 2001. 
The notion of isomorphism of triangular septuples is obvious.

Given a triangular septuple, one can construct a finite-dimensional triangular Hopf algebra $A(G, W, H, Y, B, V, u)$ as follows.

Regard $Y$ as a purely odd supervector space and consider the supergroup $H \ltimes Y$. Consider the group algebra $\mathbf{C}[H \ltimes Y]$ of this supergroup. Let $J_{V}$ be a (minimal) twist for $\mathbf{C}[H]$ corresponding to $(H, V)$ as in [EG2] (it is well defined only up to gauge equivalence). Let $J_{B}:=e^{B / 2}$, and define $\mathcal{J}:=J_{B} J_{V}$. Then $\mathcal{J}$ is a twist for $\mathbf{C}[H \ltimes Y]$, and it is clear (since $B$ is invariant) that the gauge equivalence class of $\mathcal{J}$ is independent of the choice of $J_{V}$.

Regard $W$ as an odd supervector space, and consider the supergroup $G \ltimes W$. We have a natural inclusion of supergroups $H \ltimes Y \hookrightarrow G \ltimes W$, hence $\mathcal{J}$ can be regarded as a twist for the supergroup algebra $\mathbf{C}[G \ltimes W]$. Now let $A(G, W, H, Y, B, V, u)$ be the finite-dimensional triangular Hopf algebra corresponding to the pair $\left(\mathbf{C}[G \ltimes W]^{\mathcal{J}}, u\right)$ under the correspondence of Theorem 3.3.1 of $[\mathrm{AEG}]$. According to [AEG], this Hopf algebra has the Chevalley property.

Our main result is the following theorem.

Theorem 2.2. The assignment $(G, W, H, Y, B, V, u) \rightarrow A(G, W, H, Y, B, V, u)$ is a bijection between:

1. isomorphism classes of triangular septuples, and

2. isomorphism classes of finite-dimensional triangular Hopf algebras over $\mathbf{C}$ with the Chevalley property.

Remark 2.3. It is clear that $A(G, W, H, Y, B, V, u)$ is semisimple if and only if $W=0$ (and hence $Y=0$ and $B=0$ ). Therefore, Theorem 2.2 implies, in particular, that there is a natural bijection between isomorphism classes of semisimple triangular Hopf algebras over $\mathbf{C}$, and isomorphism classes of quadruples $(G, H, V, u)$. This was the main result of [EG2]. Thus, Theorem 2.2 is a generalization of the main result of [EG2].

The rest of the section is devoted to the proof of Theorem 2.2.

We start with the following proposition, which is essentially contained in [AEG].

Proposition 2.4. Any minimal triangular Hopf superalgebra $\mathcal{A}$ over $\mathbf{C}$ with Drinfeld element 1 and the Chevalley property is isomorphic to $\mathbf{C}[H \ltimes Y]^{\mathcal{J}}$, where $H$ is a finite group, $Y$ is a finite-dimensional representation of $H$ (considered as an odd vector space) and $\mathcal{J}=e^{B / 2} J$, where $B \in\left(S^{2} Y\right)^{H}$, and $J$ is a minimal twist for $H$.

Proof. Let $\mathcal{A}_{s}:=\mathcal{A} / \operatorname{Rad}(\mathcal{A})$ be the semisimple quotient of $\mathcal{A}$. As was shown in $[\mathrm{G}]$ and $[\mathrm{AEG}]$, this is a minimal triangular semisimple Hopf algebra with Drinfeld element 1, and we have a sequence of Hopf superalgebra homomorphisms $\mathcal{A}_{s} \rightarrow \mathcal{A} \rightarrow \mathcal{A}_{s}$, with the composition being the identity. Thus, by [EG1] and [EG2], $\mathcal{A}_{s}=\mathbf{C}[H]^{J}$ with $R$-matrix $J_{21}^{-1} J$, where $H$ is a finite group, and $J$ is a minimal twist for it, corresponding to an irreducible projective representation $V$ of $H$ of dimension $|H|^{1 / 2}$. 
Let us now consider the Hopf superalgebra $\mathcal{A}^{J^{-1}}$. We have a sequence of Hopf superalgebra homomorphisms $\mathbf{C}[H] \rightarrow \mathcal{A}^{J^{-1}} \rightarrow \mathbf{C}[H]$, and the composition is the identity. The projection of the $R$-matrix $\mathcal{R}$ of $\mathcal{A}^{J^{-1}}$ to $\mathbf{C}[H] \otimes \mathbf{C}[H]$ is $1 \otimes 1$, so it is unipotent in $X \otimes Z$ for any two $\mathcal{A}$-modules $X, Z$. Let $\left(\mathcal{A}^{J^{-1}}\right)_{m}$ be the minimal part of $\mathcal{A}^{J^{-1}}$. Since any $\left(\mathcal{A}^{J^{-1}}\right)_{m}-$ module is contained in an $\mathcal{A}$-module (see e.g. [AEG]), we get that $\mathcal{R}$ is unipotent in any $X \otimes Z$, where $X, Z$ are $\left(\mathcal{A}^{J^{-1}}\right)_{m}$-modules. But this means that $\left(\mathcal{A}^{J^{-1}}\right)_{m}$ is local (since $\left(\mathcal{A}^{J^{-1}}\right)_{m} / \operatorname{Rad}\left(\left(\mathcal{A}^{J^{-1}}\right)_{m}\right)$ is minimal triangular with $R$-matrix $1 \otimes 1$, hence 1 -dimensional). Hence by [AEG], $\left(\mathcal{A}^{J^{-1}}\right)_{m}$ is $\Lambda Y$, where $Y$ is a vector space, with some triangular structure $R_{B}:=e^{B}$, where $B \in S^{2} Y$ is a nondegenerate element. Thus taking $J^{\prime}:=e^{B / 2}$, we get that $\left(\mathcal{A}^{J^{-1}}\right)^{J^{\prime-1}}$ is supercocommutative, with triangular structure $1 \otimes 1$. Then Kostant's theorem $[\mathrm{K}]$ implies that $\left(\mathcal{A}^{J^{-1}}\right)^{J^{\prime-1}}=\mathbf{C}[H \ltimes Y]$. Thus, $\mathcal{A}=\mathbf{C}[H \ltimes Y]^{J^{\prime} J}$ with triangular structure $\left(J^{\prime} J\right)_{21}^{-1} J^{\prime} J$. This concludes the proof of the proposition.

The following proposition will imply the converse of Proposition 2.4: For any $H, Y, B, J$ as above, the Hopf superalgebra $\mathcal{A}:=\mathbf{C}[H \ltimes Y]^{e^{B / 2} J}$ is minimal triangular.

Proposition 2.5. If $\left(\mathcal{A}, \mathcal{R}_{\mathcal{A}}\right)$ is a minimal triangular Hopf superalgebra, $H$ is a group acting on $\mathcal{A}$ by Hopf superalgebra automorphisms and $J$ is a minimal twist of $H$, then the Hopf superalgebra $\left((\mathbf{C}[H] \ltimes \mathcal{A})^{J}, J_{21}^{-1} \mathcal{R}_{\mathcal{A}} J\right)$ is minimal triangular.

Proof. First note that since $\mathbf{C}[H]$ is a Hopf subalgebra of $\mathbf{C}[H] \ltimes \mathcal{A}, J$ is a twist for $\mathbf{C}[H] \ltimes \mathcal{A}$, and hence $\mathcal{R}:=J_{21}^{-1} \mathcal{R}_{\mathcal{A}} J$ is a triangular structure on $\mathbf{C}[H] \ltimes \mathcal{A}$. So all we have to prove is that $\mathcal{R}$ is minimal.

Let $Z:=(\mathbf{C}[H] \ltimes \mathcal{A})_{m}$ be the minimal part, and $f:=I d \otimes \varepsilon: \mathbf{C}[H] \ltimes \mathcal{A} \rightarrow$ $\mathbf{C}[H]$. It is straightforward to check that $(I d \otimes f)(\mathcal{R})=J_{21}^{-1} J$; the minimal $R$-matrix for $\mathbf{C}[H]^{J}$. Hence $\mathbf{C}[H] \subset Z$. This implies that $\mathcal{R}_{\mathcal{A}}=J_{21} \mathcal{R} J^{-1} \in$ $Z \otimes Z$ (as $J \in Z \otimes Z$ ). So we get that $\mathcal{A} \subset Z$ as well. Since $Z$ is an algebra, we conclude that it is equal to $\mathbf{C}[H] \ltimes \mathcal{A}$ as desired.

Now let us turn to the proof of Theorem 2.2. Our job is to prove the following two statements:

Proposition 2.6. Any finite-dimensional triangular Hopf algebra $(A, R)$ over $\mathbf{C}$ with the Chevalley property is of the form $A(\mathcal{S})$ for a suitable triangular septuple $\mathcal{S}$.

Proposition 2.7. Let $A\left(\mathcal{S}_{1}\right), A\left(\mathcal{S}_{2}\right)$ be two isomorphic triangular Hopf algebras. Then the septuples $\mathcal{S}_{1}, \mathcal{S}_{2}$ are isomorphic.

Proof of Proposition 2.6. Let $(A, R)$ be a finite-dimensional triangular Hopf algebra with the Chevalley property. By [AEG], the Drinfeld element $u$ of $A$ satisfies $u^{2}=1$. Let $(\mathcal{A}, \mathcal{R})$ be the triangular Hopf superalgebra corresponding to $(A, R)$ under the correspondence given in [AEG, Theorem 3.3.1]. Recall that 
the Drinfeld element of $\mathcal{A}$ is equal to 1 , and that $u$ acts on $\mathcal{A}$ by parity. Clearly $\mathcal{A}$ has the Chevalley property as well.

Let $\mathcal{A}_{m}$ be the minimal part of $\mathcal{A}$. By Proposition 2.4, $\mathcal{A}_{m}=\mathbf{C}[H \ltimes Y]^{\mathcal{J}}$, where $\mathcal{J}=e^{B / 2} J$. Hence $\mathcal{A}^{\mathcal{J}^{-1}}$ is supercocommutative, and by Kostant's theorem $[\mathrm{K}], \mathcal{A}^{\mathcal{J}^{-1}}=\mathbf{C}[G \ltimes W]$, where $G$ is a finite group containing $H$ as a subgroup and $Y \subseteq W$ is an $H$-invariant subrepresentation. Thus we have associated to $(A, R)$ a triangular septuple $\mathcal{S}=(G, W, H, Y, B, V, u)$. It is clear that $(A, R)=A(G, W, H, Y, B, V, u)$. The proposition is proved.

Proof of Proposition 2.7. Let $\mathcal{S}_{i}=\left(G_{i}, W_{i}, H_{i}, Y_{i}, B_{i}, V_{i}, u_{i}\right), i=1,2$, be two triangular septuples, which yield isomorphic triangular Hopf algebras $A_{i}:=$ $A\left(\mathcal{S}_{i}\right)$. We want to show that $\mathcal{S}_{1}, \mathcal{S}_{2}$ are isomorphic.

Let $f: A_{1} \rightarrow A_{2}$ be an isomorphism of triangular Hopf algebras. The Drinfeld element of $A_{i}$ is $u_{i}$, so we have $f\left(u_{1}\right)=u_{2}$.

Let $\mathcal{A}_{i}$ be the Hopf superalgebra with Drinfeld element 1 corresponding to $A_{i}$ as in [AEG, Theorem 3.3.1]. Since $f\left(u_{1}\right)=u_{2}$, we find that $f$ defines an isomorphism $f: \mathcal{A}_{1} \rightarrow \mathcal{A}_{2}$ of triangular Hopf superalgebras. Thus, $f$ defines an isomorphism of their minimal parts: $f\left(\left(\mathcal{A}_{1}\right)_{m}\right)=\left(\mathcal{A}_{2}\right)_{m}$. Hence, $f\left(\operatorname{Corad}\left(\left(\mathcal{A}_{1}\right)_{m}\right)\right)=\operatorname{Corad}\left(\left(\mathcal{A}_{2}\right)_{m}\right)$. But by Proposition $2.5,\left(\mathcal{A}_{i}\right)_{m}=\mathbf{C}\left[H_{i} \ltimes\right.$

$\left.Y_{i}\right]^{e^{B_{i} / 2} J_{i}}$, so $\operatorname{Corad}\left(\left(\mathcal{A}_{i}\right)_{m}\right)=\mathbf{C}\left[H_{i}\right]^{J_{i}}$. We conclude that $f$ restricts to an isomorphism of triangular Hopf algebras $f: \mathbf{C}\left[H_{1}\right]^{J_{1}} \rightarrow \mathbf{C}\left[H_{2}\right]^{J_{2}}$. Hence, $J_{2} f^{\otimes 2}\left(J_{1}\right)^{-1}$ is a symmetric twist. So by [EG2], it is equal to $\Delta(x)\left(x^{-1} \otimes x^{-1}\right)$ for some invertible $x \in \mathbf{C}\left[H_{2}\right]$, and we have $f=\operatorname{Ad}(x) \circ \gamma$, where $\gamma$ is a group isomorphism $H_{1} \rightarrow H_{2}$.

Without loss of generality, we can assume that $x=1$ (otherwise we can apply a gauge transformation by $x$ to switch to a situation where $x=1)$. In this case, we get $f^{\otimes 2}\left(J_{1}\right)=J_{2}$, which implies $f^{*}\left(V_{1}\right)=V_{2}$, where the left hand side is the usual pullback.

Now, by definition, we have $f^{\otimes 2}\left(\left(J_{1}\right)_{21}^{-1} e^{B_{1}} J_{1}\right)=\left(J_{2}\right)_{21}^{-1} e^{B_{2}} J_{2}$. Thus, $f^{\otimes 2}\left(e^{B_{1}}\right)=e^{B_{2}}$, i.e. $f^{\otimes 2}\left(B_{1}\right)=B_{2}$. This means that $f\left(Y_{1}\right)=Y_{2}$. This map has to be consistent with the actions of $H_{i}$ on $Y_{i}$ and the map $f: H_{1} \rightarrow H_{2}$, since $f$ is a homomorphism of algebras.

Finally, setting $\mathcal{J}_{i}:=e^{B_{i} / 2} J_{i}$, we have $f^{\otimes 2}\left(\mathcal{J}_{1}\right)=\mathcal{J}_{2}$. Therefore, $f$ defines an isomorphism of triangular Hopf superalgebras $\mathbf{C}\left[G_{1} \ltimes W_{1}\right] \rightarrow \mathbf{C}\left[G_{2} \ltimes W_{2}\right]$.

Summarizing, we see that $f$ sets up an isomorphism between $\mathcal{S}_{1}$ and $\mathcal{S}_{2}$, as desired. The proposition and the theorem are proved.

\section{Minimal triangular pointed Hopf algebras}

Here we apply Theorem 2.2 to classifying minimal triangular Hopf algebras with the Chevalley property, and minimal triangular pointed Hopf algebras.

Proposition 3.1. The following hold:

1. The triangular Hopf algebra $A=A(G, W, H, Y, B, V, u)$ is minimal if and only if $Y=W$ and $G$ is generated by $H, u$. Thus, minimal triangular 
Hopf algebras over $\mathbf{C}$ with the Chevalley property correspond to triangular septuples $\mathcal{S}$ such that $W=Y$ and $G=<H, u>$.

2. The triangular Hopf algebra $A=A(G, W, H, Y, B, V, u)$ is minimal pointed if and only if $Y=W, G$ is generated by $H$ and $u$, and $G$ is abelian. Thus, minimal triangular pointed Hopf algebras over $\mathbf{C}$ correspond to triangular septuples $\mathcal{S}$ such that $W=Y, G=<H, u>$, and $G$ is abelian.

Proof. We start by proving part 1 . The "only if" direction is clear. To prove the "if" direction, consider the minimal part of the Hopf superalgebra $\mathcal{A}$ with Drinfeld element 1, corresponding to $A$. By Proposition 2.5, it is $\mathbf{C}[H \ltimes W]$ (as an algebra). The minimal part of $A$ is clearly generated by $u$ and the minimal part of $\mathcal{A}$, so it is everything.

Part 2 follows easily from part 1 , if we remember from [AEG] that any minimal triangular pointed Hopf algebra has the Chevalley property.

Remark 3.2. Proposition 3.1 implies that for minimal triangular Hopf algebras with the Chevalley property (unlike the nonminimal ones), there are finitely many isomorphism classes in any given dimension. Indeed, in a "minimal" triangular septuple, the only "continuous" parameter is the tensor $B$, or, equivalently, the $G$-invariant inner product $B^{-1}$ on $Y=W$. But such a product, if exists, is clearly unique up to an isomorphism. In particular, this implies that any minimal triangular Hopf algebra with the Chevalley property is rigid, i.e. does not have nontrivial deformations as a triangular Hopf algebra. Since it is suspected that all finite-dimensional triangular Hopf algebras over $\mathbf{C}$ have the Chevalley property (see [AEG], Question 5.5.1), it would be interesting to check whether any minimal triangular Hopf algebra is rigid.

To conclude the paper, we recall that a classification of minimal triangular pointed Hopf algebras was in fact obtained earlier in [G] (the additional condition to be generated by grouplike and skewprimitive elements imposed in $[G]$ is in fact always satisfied, as was shown in [AEG]). There, the isomorphism classes of such Hopf algebras were parameterized by somewhat different group-theoretical data than here. So, let us identify these two kinds of data with each other. This, in particular, will give another proof of Theorem 5.1 in [G], based on Theorem 2.2 .

Recall the definitions of the two types of the parameterizing data.

Type 1 (this paper): A 5 -tuple $(G, W, H, V, u)$, where $G$ is a finite abelian group, $H$ a subgroup of $G, u$ an element of $G$ of order 2, $W$ a finite-dimensional representation of $G$ with an invariant inner product, and $V$ a projective irreducible representation of $H$ of dimension $|H|^{1 / 2}$, such that $G=<H, u>$, and $\left.u\right|_{W}=-1$.

Type 2 ([G, Section 4]): A triple $(G, \varphi, n)$, where $G$ is a finite abelian group, $\varphi$ a nondegenerate skew-symmetric bilinear form on $G$ with values in $\mathbf{C}^{*}$ (i.e. $\left.\varphi(g, h)=\varphi(h, g)^{-1}\right)$, and $n: g \mapsto n_{g}$ a nonnegative integer function defined on the set $I_{\varphi}$ of elements $g \in G$ such that $\varphi(g, g)=-1$, satisfying $n_{g}=n_{g^{-1}}$ (the other data in $[\mathrm{G}]$, up to isomorphism, can be uniquely expressed via $(G, \varphi, n))$. 
Below we will describe how to pass from data of type 1 to data of type 2 , and back.

1. From Type 1 to Type 2: The group $G$ is already given, so we need to construct $\varphi$ and $n$. To construct $\varphi$, recall that the quadruple $(G, H, V, u)$ defines a minimal triangular structure on $\mathbf{C}[G]$, which is nothing but a nondegenerate skew-symmetric form on $G$; so let it be $\varphi$. Finally, to construct $n$, recall that the group $G$ is abelian, and is identified with its dual via $\varphi$, so the representation $W$ is representable as a direct sum $\oplus_{g} n_{g} \varphi(g, *)$, and we have $n_{g^{-1}}=n_{g}$ since the representation is orthogonal. Furthermore, since $\varphi(u, g)=\varphi(g, g)$, and $\left.u\right|_{W}=-1$, we get $\varphi(g, g)=-1$ if $n_{g}>0$.

2. From Type 2 to Type 1: Again, the group $G$ is given, and we need to construct the quadruple $(W, H, V, u)$. To construct $(H, V, u)$, we take the quadruple $(G, H, V, u)$ which corresponds to the triangular structure on $\mathbf{C}[G]$ defined by $\varphi$. Finally, to construct $W$, we set $W:=\oplus_{g} n_{g} \varphi(g, *)$. It is clear that this representation is orthogonal since $n_{g}=n_{g^{-1}}$, and that $u$ acts by -1 in $W$ since $\varphi(g, g)=-1$ whenever $n_{g}>0$.

It is clear that the constructed two correspondences are inverses to each other. We leave it to the reader to show that the data of type 1 and type 2 that correspond to each other under this rule, define the same minimal triangular pointed Hopf algebra.

\section{Appendix: Erratum to "Some properties and examples of pointed triangular Hopf algebras", by S. Gelaki}

In this appendix the second author wishes to report that Theorem 5.1 in the version of $[\mathrm{G}]$ which appeared in MRL is incorrect as stated; namely, the definitions of a datum $\mathcal{D}$ and the sets $\Phi$ and $S_{g}(k)$ are too restrictive and consequently there are more minimal triangular pointed Hopf algebras than claimed. However, the mistake was insignificant and, accordingly, the correction is straightforward; it follows the exact same lines of argument as in the original version. The corrected version has been posted in arxiv.org (see math.QA/9907106).

The error in the original statement and proof of Theorem 5.1 resulted from a mistake in Lemma 5.3 whose proof used a previous result of the second author, incorrectly. Namely, it is not true that if $A$ is a minimal triangular pointed Hopf algebra, then $P_{1, g}(A) \neq 0$ implies that $g$ is an involution.

We now briefly point to the main corrections made. First, the subgroup $G_{2}$ of involutions of $G$, mentioned in the paragraph preceding Definition 4.1, was replaced by the whole group $G$. That is, it is no longer assumed that for

$g \in I_{F}, g^{2}=1$. Accordingly, in Definition 4.1 the requirement $n_{g}=n_{g^{-1}}$ was added, and Formula (4) and the definitions of the sets $\Phi$ and $S_{g}(k)$, given in the discussion preceding Theorem 4.4, were adjusted. Consequently, Theorem 4.4 was generalized and its proof was adjusted. With these new definitions, the statement of Theorem 5.1 is now correct. Finally, Lemma 5.3 was corrected by 
dropping the wrong conclusion that $g$ is an involution, and Lemmas 5.4 and 5.5 were adjusted. This corrected the proof of Theorem 5.1.

\section{Acknowledgments}

The second author is grateful to MIT for its hospitality. The authors were partially supported by the NSF grant DMS-9988796. The work of the first author was done in part for the Clay Mathematics Institute.

\section{References}

[AEG] N. Andruskiewitsch, P. Etingof, and S. Gelaki, Triangular Hopf Algebras with the Chevalley Property, Michigan Math. J., to appear, math.QA/0008232.

[EG1] P. Etingof and S. Gelaki, Some Properties of Finite Dimensional Semisimple Hopf Algebras, Math. Res. Lett. 5 (1998), 191-197.

[EG2] - The Classification of Triangular Semisimple and Cosemisimple Hopf Algebras Over an Algebraically Closed Field, Internat. Math. Res. Notices 5 (2000), 223-234.

[G] S. Gelaki, Some Properties and Examples of Pointed Triangular Hopf Algebras, Math. Res. Lett. 6 (1999), 563-572; see corrected version at math.QA/9907106.

[K] B. Kostant, Graded manifolds, graded Lie theory, and prequantization, Differential geometrical methods in mathematical physics, Proc. Symp. Bonn 1975, Lecture Notes in Math. 570 (1977), 177-306.

Department of Mathematics, Massachusetts Institute of Technology, 77 MasSachusetts Avenue, Cambridge, MA 02139.

Department of Mathematics, Columbia University, 2990 Broadway, New York, Ny 10027.

E-mail address: etingof@math.mit.edu

Department of Mathematics, Technion-Israel Institute of Technology, Haifa 32000, ISRAEL.

E-mail address: gelaki@math.technion.ac.il 